\title{
Synthesis and study of new $2 H$-pyranoquinolin-2-one-based inhibitors of blood coagulation factors Xa and XIa
}

\author{
A. Yu. Potapov, ${ }^{a \star}$ B. V. Paponov, ${ }^{b}$ N. A. Podoplelova, ${ }^{c}$ M. A. Panteleev, ${ }^{c}$ V. A. Polikarchuk, ${ }^{a}$ \\ I. V. Ledenyova, ${ }^{a}$ N. V. Stolpovskaya, ${ }^{a} D$. V. Kryl'skii, ${ }^{d}$ and Kh. S. Shikhaliev ${ }^{a}$ \\ ${ }^{a}$ Voronezh State University, \\ 1 Universitetskaya pl., 394018 Voronezh, Russian Federation. \\ Fax: +7 (473) 255 6890. E-mail: pistones@mail.ru \\ ${ }^{b}$ Belgorod State National Research University, \\ 85 ul. Pobedy, 308015 Belgorod, Russian Federation \\ ${ }^{c}$ Center for Theoretical Problems of Physicochemical Pharmacology, Russian Academy of Sciences, \\ 30 ul. Srednyaya Kalitnikovskaya, 109029 Moscow, Russian Federation \\ ${ }^{d}$ Research Institute of Applied Acoustics, \\ 7A ul. 9 Maya, 141980 Dubna, Moscow Region, Russian Federation
}

\begin{abstract}
Condensation of 7-hydroxy-1,2,2,4-tetramethylhydroquinoline-6-carbaldehydes with various hetaryl-substituted 3-oxo esters afforded a series of hybrid hydro-6,8,8,9-tetramethyl$2 \mathrm{H}$-pyrano[3,2-g]quinolin-2-ones. A number of these compounds exhibited relatively high inhibitory activity against blood coagulation factors Xa and XIa.
\end{abstract}

Key words: blood coagulation factor Xa, blood coagulation factor XIa, molecular hybridization, 7-hydroxyhydroquinoline-6-carbaldehyde, methyl pyrimidine-3-oxopropionate, $2 H$-pyrano[3,2-g]quinolin-2-one.

It is known that existing antithrombotic drugs can cause uncontrolled bleeding, therefore, researchers face an issue related to the development of new inhibitors of blood coagulation factors that do not have this disadvantage. At present, there are developed and introduced into clinical practice $^{1-3}$ direct inhibitors of blood coagulation enzymes, namely, the serine protease factor Xa (FXa), one of the key components of thrombus formation. ${ }^{4-7}$ The developed inhibitors of blood coagulation factor XIa (FXIa) are undergoing laboratory and clinical trials. ${ }^{8-14}$ However, due to the presence of structurally similar binding sites for serine protease factor and thrombin, ${ }^{15,16}$ the search for new selective inhibitors of FXa and FXIa is an actual problem.

For a long time, coumarin derivatives, being the vitamin $\mathrm{K}$ antagonists, were the only oral anticoagulants. At present, a number of coumarin derivatives have been identified as exhibiting high inhibitory activity against FXa and FXIa. ${ }^{14,17-20}$ In addition, it was found that derivatives of dihydro- ${ }^{21}$ and tetrahydroquinolines, $\mathbf{9 0 , 1 8 , 2 2 - 2 3}$ exhibit similar biological activity.

In the framework of a rational and efficient design of biologically active compounds, researchers widely use the method of molecular hybridization, which is based on the combination of several pharmacophore fragments in one molecule. ${ }^{24-32}$ The implementation of such an approach in order to search for new highly efficient inhibitors of blood coagulation factors Xa and XIa based on the hybridization of hydroquinoline $(\boldsymbol{A})$ and coumarin $(\boldsymbol{B})$ rings seems to be very promising. $2 H$-Pyrano[3,2-g]quinolinones exemplify this type of hybrid molecules.

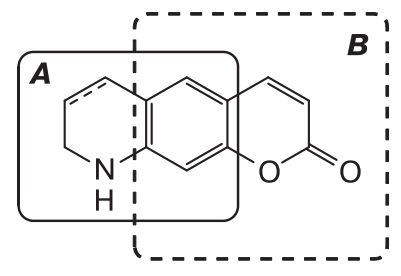

$2 H$-Pyrano[3,2-g]quinolinones

The purpose of the present work was to obtain new substituted pyrano[3,2-g]quinolin-2-one systems with various degrees of hydrogenation and to study their inhibitory activity against FXa and FXIa.

It is known that Knoevenagel condensation of salicylic aldehyde derivatives with methylene active carbonyl compounds leads to coumarins ( $2 H$-chromen-2-ones). ${ }^{33-36}$ We found that the condensation of 7-hydroxy-1,2,2,4tetramethylhydroquinoline-6-carbaldehydes $\mathbf{1}$ and $\mathbf{2}$ with methyl 2-R-4-methyl-5-pyrimidine-3-oxopropanoates $3 \mathbf{a}-\mathbf{e}$ under the conditions we described earlier ${ }^{37}$ gives 8,9-dihydro-2 $H$-pyrano[3,2-g] quinolin-2-ones $\mathbf{4 a - c}$ and 
6,7,8,9-tetrahydro-2 $\mathrm{H}$-pyrano[3,2-g]quinolin-2-ones 5a-e in fairly high yields $(70-93 \%)$ (Scheme 1).

\section{Scheme 1}<smiles>[R]c1ncc(C(=O)CC(=O)OCC)c(C)n1</smiles><smiles>[R]c1ncc(C(=O)c2cc3cc4c(cc3oc2=O)N(C)C(C)(C)C=C4C)c(C)n1</smiles>

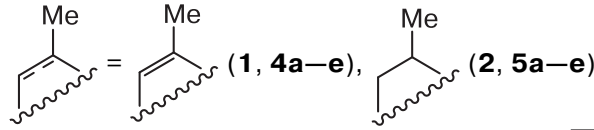

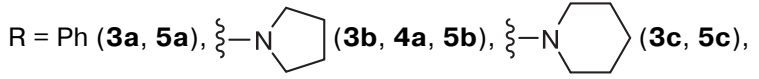

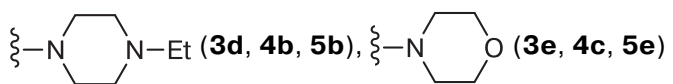

Reagents and conditions: EtOH, piperidine, reflux, $2 \mathrm{~h}$.

A plausible route of this reaction is shown in Scheme 2. The dehydration of the Knoevenagel adducts $\mathbf{A}$ formed from aldehydes $\mathbf{1}$ or $\mathbf{2}$ and keto esters $\mathbf{3}$ leads to arylidene derivatives $\mathbf{B}$, the intramolecular cyclization of which gives the final products $\mathbf{4}$ and $\mathbf{5}$.

In order to expand the combinatorial library of $2 \mathrm{H}$-pyrano[3,2-g]quinolinones, we involved 4-hetarylthioacetoacetic esters $\mathbf{6 a}, \mathbf{b}$ in the reaction described above. It was found that the condensation of hydroxycarbaldehydes $\mathbf{1}$ and $\mathbf{2}$ with esters $\mathbf{6 a}, \mathbf{b}$ proceeds smoothly in refluxing ethanol in the presence of piperidine and leads to hetarylthio-8,9-dihydro-2 $H$-pyrano[3,2-g]quinolin-2-ones 7a,b and hetarylthio-6,7,8,9-tetrahydro- $2 H$-pyrano[3,2-g]quinolin-2-ones 8a,b (Scheme 3). Possible side condensation of the carbonyl component with the $\mathrm{CH}$-active mercaptomethylene group did not occur, and the target products $\mathbf{7 a , b}$ and $\mathbf{8 a , b}$ were isolated in high yields (79-84\%).

All the synthesized compounds are chromatographically pure crystalline substances identified by ${ }^{1} \mathrm{H}$ and ${ }^{13} \mathrm{C}$ NMR spectroscopy and high-resolution mass spectrometry.

The ${ }^{1} \mathrm{H}$ NMR spectra of compounds $\mathbf{4 a}-\mathbf{c}, \mathbf{5} \mathbf{a}-\mathbf{e}$, $\mathbf{7 a}, \mathbf{b}$, and $\mathbf{8 a}, \mathbf{b}$, in contrast to the spectra of the starting hydroxyquinolinecarbaldehydes $\mathbf{1}$ and $\mathbf{2}$, do not exhibit signals for the protons of the hydroxy and aldehyde groups. Their spectra exhibit signals for the aromatic protons of the tricyclic framework in the regions of $\delta 6.40-6.43$ $(\mathrm{H}(10)), 7.38-7.43(\mathrm{H}(5))$, and 8.23-8.52 $(\mathrm{H}(4))$. The signals for the protons $\mathrm{H}(7)$ of 8,9-dihydro derivatives $\mathbf{4 a - c}$ and $\mathbf{7 a , b}$ are observed as singlets in the region of $\delta 5.48-5.51$. In the case of 6,7,8,9-tetrahydro derivatives $\mathbf{5 a}-\mathbf{e}$ and $\mathbf{8 a}, \mathbf{b}$, the two protons at position 7 (protons $\mathrm{A}$ and $\mathrm{M}$ ) and the proton in position 6 (proton $\mathrm{X}$ ) form a three-spin system AMX, with the proton $\mathrm{X}$ being additionally coupled with the protons of the methyl group at position 6 of the tricyclic system. The signals for the protons $\mathrm{A}$ of the AMX system are observed in the region of

Scheme 2<smiles>[R]C(=O)CC(=O)OCC</smiles><smiles>[R]C(=O)C(C(=O)OCC)C(O)c1cc2c(cc1O)N(C)C(C)(C)C=C2C</smiles><smiles>CC1=CC(C)(C)N(C)c2cc3oc(=O)c(C(=O)F)cc3cc21</smiles><smiles>CC</smiles><smiles>[R]C(=O)/C(=C/C(=O)OCC)c1cc2c(cc1O)N(C)C(C)(C)C=C2C</smiles> 
Scheme 3<smiles>CCOC(=O)CC(=O)CSc1ccccc1</smiles><smiles>CCC(C)(C)OC(=O)CSc1ccccc1</smiles>

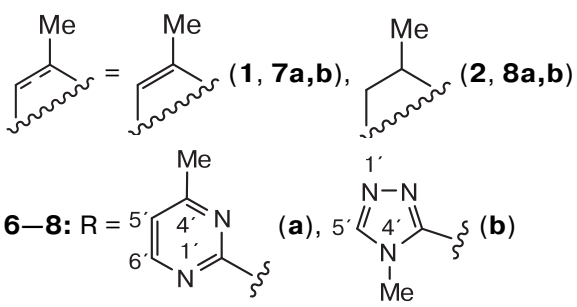

Reagents and conditions: EtOH, piperidine, reflux, 2 h.

$\delta 1.39-1.41$ as triplets $\left(J_{\mathrm{AM}}=J_{\mathrm{AX}}=13.0-13.2 \mathrm{~Hz}\right)$ with an integral intensity of $1 \mathrm{H}$. The signals for protons $\mathrm{M}$ are found at about $\delta 1.87$ as doublets of doublets $\left(J_{\mathrm{AM}}=\right.$ $=13.0-13.2 \mathrm{~Hz}, J_{\mathrm{MX}}=3.9-4.0 \mathrm{~Hz}$ ) with an integral intensity of $1 \mathrm{H}$. The protons $\mathrm{X}$ resonate at about $\delta 2.77$ as a poorly resolved septet with an integral intensity of $1 \mathrm{H}$; it was impossible in this case to correctly determine the spin-spin coupling constants. In the ${ }^{13} \mathrm{C}$ NMR spectra of the synthesized compounds, the signals for the carbon atoms of the keto group at position 3 of the tricyclic system are observed at $\delta 189-190$.

We carried out preliminary in vitro screening of compounds $\mathbf{4 a - c}, \mathbf{5 a}-\mathbf{e}, \mathbf{7 a}, \mathbf{b}$, and $\mathbf{8 a}, \mathbf{b}$ in order to identify the lead compounds and determine their relative inhibitory activity against factors Xa and XIa. A reference drug was the clinically used oral anticoagulant rivaroxaban, which selectively inhibits factor $\mathrm{Xa}$ and has almost no effect on factor XIa. It was found that compounds $\mathbf{5 a}$ and $\mathbf{5 c}$ containing a phenyl and a piperidine substituent in the pyrimidine ring have the greatest inhibitory effect on factors Xa and XIa. Compounds $\mathbf{4 a}$ and $\mathbf{7 a}$ moderately inhibited factor XIa and slightly inhibited factor Xa (Table 1). Compounds $\mathbf{4 b , c}$ and $\mathbf{7 b}$ slightly activated factors Xa and XIa.

The obtained results form the basis for further studies of the possibilities of the molecular hybridization method in order to search for highly efficient and selective inhibitors of blood coagulation factors among hydroquinoline derivatives.
Table 1. Inhibitory activity of compounds $4 \mathbf{a}-\mathbf{c}, \mathbf{5 a}-\mathbf{e}, \mathbf{7 a}, \mathbf{b}$, $\mathbf{8 a}, \mathbf{b}$ and rivaroxaban against blood coagulation factors $\mathrm{Xa}$ and XIa

\begin{tabular}{lcc}
\hline Compound & \multicolumn{2}{c}{ Factor activity (in \% to control*) } \\
\cline { 2 - 3 } & Xa & XIa \\
\hline 4a & 67 & 14 \\
$\mathbf{4 b}$ & 122 & 108 \\
4c & 104 & 42 \\
5a & 14 & 7 \\
5b & 86 & 83 \\
5c & 11 & 5 \\
5d & 96 & 81 \\
5e & 98 & 81 \\
7a & 97 & 10 \\
7b & 99 & 102 \\
8a & 80 & 85 \\
8b & 95 & 99 \\
Rivaroxaban & 6 & 92 \\
\hline
\end{tabular}

* The control sample did not contain the test compounds.

\section{Experimental}

${ }^{1} \mathrm{H}$ and ${ }^{13} \mathrm{C}$ NMR spectra were recorded on an Agilent MR 400+ spectrometer (400 and $100 \mathrm{MHz}$, respectively) in DMSO- $\mathrm{d}_{6}$ under normal conditions. High-resolution electrospray ionization (ESI) mass spectra were obtained on an Agilent Technologies 1260 infinity instrument with an Agilent 6230 TOF LC/MS time-of-flight mass detector in the positive ion mode (the nebulizer gas $\left(\mathrm{N}_{2}\right)$ pressure $20 \mathrm{psi}$, the drying gas flow rate $6 \mathrm{~mL} \mathrm{~min}-1$, the gas temperature $325^{\circ} \mathrm{C}$; the capillary voltage $4.0 \mathrm{kV}$, the fragmentator voltage $+191 \mathrm{~V}$, the skimmer voltage $+66 \mathrm{~V}$, the OctRF parameter $750 \mathrm{~V}$; the mass detection range 50-2000 Da; a Poroshell 120 EC-C18 column $(4.6 \times 50 \mathrm{~mm}$, $2.7 \mu \mathrm{m})$, a gradient elution with a mixture of acetonitrile-water containing $0.1 \%$ of formic acid (the $\mathrm{MeCN}$ gradient from $60 \%$ to $98 \%$ over $3 \mathrm{~min}$, the eluent flow rate $0.4 \mathrm{~mL} \mathrm{~min}^{-1}$ )). The results were processed using the MassHunter Workstation/Data Acquisition V.06.00 software. Melting points were measured using a Stuart SMP30 apparatus. Reaction progress, purity of the starting compounds and products, as well as the qualitative analysis of the reaction mixtures, were monitored by TLC on Merck TLC Silica gel $60 \mathrm{~F}_{254}$ plates (eluents chloroform, methanol, and their mixtures in various ratios). The spots of compounds were visualized in UV light or iodine vapors.

7-Hydroxy-1,2,2,4-tetramethylhydroquinoline-6-carbaldehydes 1 and 2 were obtained according to the procedures described in the works. ${ }^{37,38}$ The starting methyl 2-R-4-methyl-5pyrimidine-3-oxopropionates $\mathbf{3 a}-\mathbf{e}$ and 4-hetarylthioacetoacetic esters $\mathbf{6} \mathbf{a}, \mathbf{b}$ were purchased from Alinda Chemical Ltd.

Synthesis of 3-(2-R-pyrimidine-5-carbonyl)-6,8,8,9-tetramethyl-2H-pyrano[3,2-g]quinolin-2-ones $(4 a-c, 5 a-e, 7 a, b$, and 8a,b) (general procedure). A mixture of carbaldehyde $\mathbf{1}$ or $\mathbf{2}$ $(0.002 \mathrm{~mol})$, the corresponding ester $\mathbf{3 a}-\mathbf{e}$ or $\mathbf{6 a}, \mathbf{b}(0.002 \mathrm{~mol})$, ethanol $(15 \mathrm{~mL})$, and two-three drops of piperidine was refluxed for $2 \mathrm{~h}$. The precipitate formed upon cooling the reaction mixture was collected by filtration and recrystallized from a mixture of EtOH-DMF. 
3-[4-Methyl-2-(pyrrolidin-1-yl)pyrimidine-5-carbonyl]6,8,8,9-tetramethyl-8,9-dihydro-2 $H$-pyrano[3,2-g]quinolin-2one (4a). The yield was $0.74 \mathrm{~g}(83 \%)$, m.p. $178-179^{\circ} \mathrm{C}$. ${ }^{1} \mathrm{H}$ NMR, $\delta: 1.36\left(\mathrm{~s}, 6 \mathrm{H}, \mathrm{C}(8)\left(\mathrm{CH}_{3}\right)_{2}\right) ; 1.82-1.91\left(\mathrm{~m}, 7 \mathrm{H}, 2 \mathrm{NCH}_{2} \mathrm{CH}_{2}\right.$ of pyrrolidine, $\left.\mathrm{C}(6) \mathrm{CH}_{3}\right) ; 2.42$ (s, $\left.3 \mathrm{H}, \mathrm{N}(9) \mathrm{CH}_{3}\right) ; 2.90(\mathrm{~s}, 3 \mathrm{H}$, $\left.\mathrm{C}\left(4^{\prime}\right) \mathrm{CH}_{3}\right) ; 3.53$ (br.s, $4 \mathrm{H}, 2 \mathrm{NCH}_{2}$ of pyrrolidine); 5.48 (s, $1 \mathrm{H}$, $\mathrm{H}(7)) ; 6.40$ (s, $1 \mathrm{H}, \mathrm{H}(10)) ; 7.38$ (s, $1 \mathrm{H}, \mathrm{H}(5)) ; 8.23$ (s, $1 \mathrm{H}$, $\mathrm{H}(4)) ; 8.39$ (s, $\left.1 \mathrm{H}, \mathrm{H}\left(2^{\prime}\right)\right)$ ). ${ }^{13} \mathrm{C}$ NMR, $\delta: 18.6 ; 24.3 ; 25.3 ; 29.1$; $29.1 ; 47.0 ; 58.4 ; 95.9 ; 108.1 ; 118.0 ; 120.0 ; 120.2 ; 124.4 ; 125.7$; $130.6 ; 148.2 ; 150.9 ; 158.5 ; 159.2 ; 159.6 ; 160.8 ; 168.0 ; 189.9$. MS, found $m / z 445.2237[\mathrm{M}+\mathrm{H}]^{+}$, calculated for $\mathrm{C}_{26} \mathrm{H}_{28} \mathrm{~N}_{4} \mathrm{O}_{3}$ : 445.2236 .

3-[2-(4-Ethylpiperazin-1-yl)-4-methylpyrimidine-5-carbonyl]-6,8,8,9-tetramethyl-8,9-dihydro- $2 \mathrm{H}$-pyrano [3,2-g]quinolin-2-one (4b). The yield was $0.69 \mathrm{~g} \mathrm{(71 \% ),} \mathrm{m.p.} \mathrm{193-194}{ }^{\circ} \mathrm{C}$. ${ }^{1} \mathrm{H}$ NMR, $\delta: 1.01\left(\mathrm{t}, 3 \mathrm{H}, \mathrm{CH}_{2} \mathrm{CH}_{3}, J=7.1 \mathrm{~Hz}\right) ; 1.35(\mathrm{~s}, 6 \mathrm{H}$, $\left.\mathrm{C}(8)\left(\mathrm{CH}_{3}\right)_{2}\right) ; 1.93\left(\mathrm{~s}, 3 \mathrm{H}, \mathrm{C}(6) \mathrm{CH}_{3}\right) ; 2.35\left(\mathrm{q}, 2 \mathrm{H}, \mathrm{CH}_{2} \mathrm{CH}_{3}\right.$, $J=7.1 \mathrm{~Hz}) ; 2.38-2.42\left(\mathrm{~m}, 7 \mathrm{H}, 2 \mathrm{NCH}_{2} \mathrm{CH}_{2} \mathrm{NH}, \mathrm{N}(9) \mathrm{CH}_{3}\right)$; 2.90 (s, $3 \mathrm{H}, \mathrm{C}\left(4^{\prime}\right) \mathrm{CH}_{3}$ ); 3.81 (br.s, $\left.4 \mathrm{H}, 2 \mathrm{NCH}_{2} \mathrm{CH}_{2} \mathrm{NH}\right)$; 5.48 (s, $1 \mathrm{H}, \mathrm{H}(7))$; 6.40 (s, $1 \mathrm{H}, \mathrm{H}(10)) ; 7.38$ (s, $1 \mathrm{H}, \mathrm{H}(5))$; 8.25 (s, $1 \mathrm{H}, \mathrm{H}(4)) ; 8.40$ (s, $\left.1 \mathrm{H}, \mathrm{H}\left(2^{\prime}\right)\right) .{ }^{13} \mathrm{C}$ NMR, $\delta: 12.4$; 18.6 ; $24.2 ; 29.1 ; 32.2 ; 43.8 ; 52.0 ; 52.6 ; 58.4 ; 95.9 ; 108.2 ; 117.7$; $120.1 ; 120.8 ; 124.4 ; 125.7 ; 130.6 ; 148.4 ; 151.1 ; 158.5 ; 159.5$; 160.3 ; 160.8; 168.1; 189.6. MS, found: $m / z 488.2656[\mathrm{M}+\mathrm{H}]^{+}$, calculated for $\mathrm{C}_{28} \mathrm{H}_{33} \mathrm{~N}_{5} \mathrm{O}_{3}: 488.2658$.

3-[4-Methyl-2-(morpholin-4-yl)pyrimidine-5-carbonyl]6,8,8,9-tetramethyl-8,9-dihydro- $2 H$-pyrano[3,2-g]quinolin-2one (4c). The yield was $0.73 \mathrm{~g}(79 \%)$, m.p. $227-228^{\circ} \mathrm{C} .{ }^{1} \mathrm{H}$ NMR, $\delta: 1.36\left(\mathrm{~s}, 6 \mathrm{H}, \mathrm{C}(8)\left(\mathrm{CH}_{3}\right)_{2}\right) ; 1.93\left(\mathrm{~s}, 3 \mathrm{H}, \mathrm{C}(6) \mathrm{CH}_{3}\right) ; 2.42$ (s, $3 \mathrm{H}$, $\left.\mathrm{N}(9) \mathrm{CH}_{3}\right) ; 2.91\left(\mathrm{~s}, 3 \mathrm{H}, \mathrm{C}\left(4^{\prime}\right) \mathrm{CH}_{3}\right) ; 3.65\left(\mathrm{t}, 4 \mathrm{H}, 2 \mathrm{NCH}_{2} \mathrm{CH}_{2} \mathrm{O}\right.$, $J=5.1 \mathrm{~Hz}) ; 3.81\left(\mathrm{t}, 4 \mathrm{H}, 2 \mathrm{NCH}_{2} \mathrm{CH}_{2} \mathrm{O}, J=5.1 \mathrm{~Hz}\right) ; 5.49$ (s, $1 \mathrm{H}, \mathrm{H}(7)) ; 6.42$ (s, $1 \mathrm{H}, \mathrm{H}(10)$ ); 7.40 (s, $1 \mathrm{H}, \mathrm{H}(5)$ ); 8.28 (s, $1 \mathrm{H}, \mathrm{H}(4)) ; 8.43$ (s, $\left.1 \mathrm{H}, \mathrm{H}\left(2^{\prime}\right)\right) .{ }^{13} \mathrm{C}$ NMR, $\delta: 18.8 ; 24.2 ; 29.1$; $32.3 ; 44.2 ; 58.5 ; 66.4 ; 95.9 ; 108.2 ; 117.5 ; 119.7 ; 121.2 ; 124.5$; $125.3 ; 130.6 ; 148.6 ; 151.1 ; 158.6 ; 159.6 ; 160.4 ; 160.6 ; 168.0$; 189.7. MS, found: $m / z 461.2185[\mathrm{M}+\mathrm{H}]^{+}$, calculated for $\mathrm{C}_{26} \mathrm{H}_{28} \mathrm{~N}_{4} \mathrm{O}_{4}: 461.2185$.

3-[4-Methyl-2-phenylpyrimidine-5-carbonyl]-6,8,8,9-tetramethyl-6,7,8,9-tetrahydro-2H-pyrano[3,2-g]quinolin-2-one (5a). The yield was $0.65 \mathrm{~g}(72 \%)$, m.p. $191-192{ }^{\circ} \mathrm{C} .{ }^{1} \mathrm{H}$ NMR, $\delta: 1.26$ (s, $\left.3 \mathrm{H}, \mathrm{C}(8) \mathrm{CH}_{3}\right) ; 1.28-1.32\left(\mathrm{~m}, 6 \mathrm{H}, \mathrm{C}(6) \mathrm{CH}_{3}, \mathrm{C}(8) \mathrm{CH}_{3}\right)$; $1.40(\mathrm{t}, 1 \mathrm{H}, \mathrm{H}(7), J=13.0 \mathrm{~Hz}) ; 1.88(\mathrm{dd}, 1 \mathrm{H}, \mathrm{H}(7), J=13.0 \mathrm{~Hz}$, $J=3.9 \mathrm{~Hz}) ; 2.52\left(\mathrm{~s}, 3 \mathrm{H}, \mathrm{N}(9) \mathrm{CH}_{3}\right) ; 2.77$ (m, $\left.1 \mathrm{H}, \mathrm{H}(6)\right) ; 2.93$ (s, $\left.3 \mathrm{H}, \mathrm{C}\left(4^{\prime}\right) \mathrm{CH}_{3}\right) ; 6.47$ (s, $\left.1 \mathrm{H}, \mathrm{H}(10)\right) ; 7.51-7.57$ (m, $4 \mathrm{H}$, $\mathrm{Ph}) ; 8.42-8.44$ (m, $\left.2 \mathrm{H}, p-\mathrm{H}_{\mathrm{Ph}}, \mathrm{H}(5)\right) ; 8.56$ (s, $\left.1 \mathrm{H}, \mathrm{H}(4)\right) ; 8.79$ (s, $\left.1 \mathrm{H}, \mathrm{H}\left(2^{\prime}\right)\right) .{ }^{13} \mathrm{C}$ NMR, $\delta: 19.3 ; 23.1 ; 25.5 ; 26.6 ; 28.9 ; 32.9$; $45.1 ; 56.6 ; 96.5 ; 108.2 ; 114.8 ; 127.3 ; 128.4 ; 129.2 ; 131.7 ; 132.1$; $137.1 ; 149.9 ; 153.1 ; 156.4 ; 158.0 ; 159.8 ; 163.1 ; 164.7 ; 190.5$. MS, found: $m / z 454.2125[\mathrm{M}+\mathrm{H}]^{+}$, calculated for $\mathrm{C}_{28} \mathrm{H}_{27} \mathrm{~N}_{3} \mathrm{O}_{3}$ : 524.2127 .

3-[4-Methyl-2-(pyrrolidin-1-yl)pyrimidine-5-carbonyl]6,8,8,9-tetramethyl-6,7,8,9-tetrahydro- $2 \mathrm{H}$-pyrano[2,3-g]quinolin-2-one (5b). The yield was $0.80 \mathrm{~g}(90 \%)$, m.p. $141-145^{\circ} \mathrm{C}$. ${ }^{1} \mathrm{H}$ NMR, $\delta: 1.25$ (s, $\left.3 \mathrm{H}, \mathrm{C}(8) \mathrm{CH}_{3}\right) ; 1.28-1.32\left(\mathrm{~m}, 6 \mathrm{H}, \mathrm{C}(6) \mathrm{CH}_{3}\right.$, $\left.\mathrm{C}(8) \mathrm{CH}_{3}\right) ; 1.40(\mathrm{t}, 1 \mathrm{H}, \mathrm{H}(7), J=13.1 \mathrm{~Hz}) ; 1.88(\mathrm{dd}, 1 \mathrm{H}, \mathrm{H}(7)$, $J=13.1 \mathrm{~Hz}, J=4.0 \mathrm{~Hz}) ; 1.91-1.94\left(\mathrm{~m}, 4 \mathrm{H}, 2 \mathrm{NCH}_{2} \mathrm{CH}_{2}\right.$ of pyrrolidine); 2.42 (s, $\left.3 \mathrm{H}, \mathrm{N}(9) \mathrm{CH}_{3}\right) ; 2.78(\mathrm{~m}, 1 \mathrm{H}, \mathrm{H}(6)) ; 2.91$ (s, $\left.3 \mathrm{H}, \mathrm{C}\left(4^{\prime}\right) \mathrm{CH}_{3}\right) ; 3.54$ (br.s, $4 \mathrm{H}, 2 \mathrm{NCH}_{2}$ of pyrrolidine); 6.45 (s, $1 \mathrm{H}, \mathrm{H}(10)) ; 7.48$ (s, $1 \mathrm{H}, \mathrm{H}(5)) ; 8.26$ (s, $1 \mathrm{H}, \mathrm{H}(4))$; 8.37 (s, $\left.1 \mathrm{H}, \mathrm{H}\left(2^{\prime}\right)\right) .{ }^{13} \mathrm{C} \mathrm{NMR}, \delta: 19.4 ; 24.2 ; 25.3 ; 25.5 ; 26.7$; $29.0 ; 32.7 ; 45.4 ; 47.0 ; 56.2 ; 96.5 ; 107.8 ; 117.8 ; 120.4 ; 126.4$;
$126.9 ; 148.3 ; 152.0 ; 157.1 ; 159.3 ; 159.8 ; 160.7 ; 160.9 ; 190.0 . \mathrm{MS}$, found: $m / z 447.2391[\mathrm{M}+\mathrm{H}]^{+}$, calculated for $\mathrm{C}_{26} \mathrm{H}_{30} \mathrm{~N}_{4} \mathrm{O}_{3}$ : 447.2392 .

3-[4-Methyl-2-(piperidin-1-yl)pyrimidine-5-carbonyl]6,8,8,9-tetramethyl-6,7,8,9-tetrahydro- $2 \mathrm{H}$-pyrano[3,2-g]quinolin-2-one (5c). The yield was $0.70 \mathrm{~g}(76 \%)$, m.p. $165-170{ }^{\circ} \mathrm{C}$. ${ }^{1} \mathrm{H}$ NMR, $\delta: 1.25\left(\mathrm{~s}, 3 \mathrm{H}, \mathrm{C}(8) \mathrm{CH}_{3}\right) ; 1.29-1.33\left(\mathrm{~m}, 6 \mathrm{H}, \mathrm{C}(6) \mathrm{CH}_{3}\right.$, $\left.\mathrm{C}(8) \mathrm{CH}_{3}\right) ; 1.40(\mathrm{t}, 1 \mathrm{H}, \mathrm{H}(7), J=13.2 \mathrm{~Hz}) ; 1.49-1.56(\mathrm{~m}, 4 \mathrm{H}$, $2 \mathrm{NCH}_{2} \mathrm{CH}_{2}$ of piperidine); 1.61-1.68 (m, $2 \mathrm{H}, \mathrm{NCH}_{2} \mathrm{CH}_{2} \mathrm{CH}_{2}$ of piperidine); $1.87(\mathrm{dd}, 1 \mathrm{H}, \mathrm{H}(7), J=13.2 \mathrm{~Hz}, J=4.0 \mathrm{~Hz})$; $2.41\left(\mathrm{~s}, 3 \mathrm{H}, \mathrm{N}(9) \mathrm{CH}_{3}\right) ; 2.78(\mathrm{~m}, 1 \mathrm{H}, \mathrm{H}(6)) ; 2.91(\mathrm{~s}, 3 \mathrm{H}$, $\left.\mathrm{C}\left(4^{\prime}\right) \mathrm{CH}_{3}\right) ; 3.71-3.77\left(\mathrm{~m}, 4 \mathrm{H}, 2 \mathrm{NCH}_{2}\right.$ of piperidine $) ; 6.45$ (s, $1 \mathrm{H}, \mathrm{H}(10)) ; 7.48$ (s, $1 \mathrm{H}, \mathrm{H}(5)) ; 8.27$ (s, $1 \mathrm{H}, \mathrm{H}(4)) ; 8.37$ (s, $\left.1 \mathrm{H}, \mathrm{H}\left(2^{\prime}\right)\right) .{ }^{13} \mathrm{C}$ NMR, $\delta: 19.4 ; 24.3 ; 24.6 ; 25.5 ; 25.9 ; 26.7 ; 29.0$; 32.7 ; 44.6; 45.4; $56.2 ; 96.5 ; 107.7 ; 117.7 ; 120.3 ; 126.4 ; 127.1$; $148.4 ; 152.0 ; 157.1 ; 159.8 ; 160.2 ; 160.9 ; 168.1 ; 189.7$. MS, found: $m / z 461.2546[\mathrm{M}+\mathrm{H}]^{+}$, calculated for $\mathrm{C}_{27} \mathrm{H}_{32} \mathrm{~N}_{4} \mathrm{O}_{3}: 461.2549$.

3-[2-(4-Ethylpiperazin-1-yl)-4-methylpyrimidine-5-carbonyl]-6,8,8,9-tetramethyl-6,7,8,9-tetrahydro- $2 \mathrm{H}$-pyrano[3,2-g]quinolin-2-one (5d). The yield was $0.87 \mathrm{~g}(89 \%)$, m.p. 199$200{ }^{\circ} \mathrm{C} .{ }^{1} \mathrm{H}$ NMR, $\delta: 1.01$ (t, $\left.3 \mathrm{H}, \mathrm{CH}_{2} \mathrm{CH}_{3}, J=14.4 \mathrm{~Hz}\right) ; 1.24$ $\left(\mathrm{s}, 3 \mathrm{H}, \mathrm{C}(8) \mathrm{CH}_{3}\right) ; 1.29-1.32\left(\mathrm{~m}, 6 \mathrm{H}, \mathrm{C}(6) \mathrm{CH}_{3}, \mathrm{C}(8) \mathrm{CH}_{3}\right)$; $1.39(\mathrm{t}, 1 \mathrm{H}, \mathrm{H}(7), J=13.1 \mathrm{~Hz}) ; 1.87(\mathrm{dd}, 1 \mathrm{H}, \mathrm{H}(7), J=13.1 \mathrm{~Hz}$, $J=4.0 \mathrm{~Hz}) ; 2.34\left(\mathrm{q}, 2 \mathrm{H}, \mathrm{CH}_{2} \mathrm{CH}_{3}, J=14.4 \mathrm{~Hz}\right) ; 2.39-2.41$ (m, $\left.7 \mathrm{H}, 2 \mathrm{NCH}_{2} \mathrm{CH}_{2} \mathrm{NH}, \mathrm{N}(9) \mathrm{CH}_{3}\right) ; 2.77$ (m, $\left.1 \mathrm{H}, \mathrm{H}(6)\right) ; 2.91$ (s, $\left.3 \mathrm{H}, \mathrm{C}\left(4^{\prime}\right) \mathrm{CH}_{3}\right) ; 3.81$ (br.s, $4 \mathrm{H}, 2 \mathrm{NC}_{2} \mathrm{CH}_{2} \mathrm{NH}$ ); 6.44 (s, $1 \mathrm{H}, \mathrm{H}(10)) ; 7.47$ (s, $1 \mathrm{H}, \mathrm{H}(5)) ; 8.27$ (s, $1 \mathrm{H}, \mathrm{H}(4)) ; 8.37$ (s, $\left.1 \mathrm{H}, \mathrm{H}\left(2^{\prime}\right)\right) .{ }^{13} \mathrm{C}$ NMR, $\delta: 12.4 ; 19.4 ; 24.1 ; 25.5 ; 26.7 ; 28.9$; $32.7 ; 43.8 ; 45.4 ; 52.0 ; 52.6 ; 56.2 ; 96.5 ; 107.8 ; 117.5 ; 121.0 ; 126.5$; $126.9 ; 148.5 ; 152.1 ; 157.2 ; 159.8 ; 160.4 ; 160.6 ; 168.0 ; 189.8 . \mathrm{MS}$, found: $m / z 490.2810[\mathrm{M}+\mathrm{H}]^{+}$, calculated for $\mathrm{C}_{28} \mathrm{H}_{35} \mathrm{~N}_{5} \mathrm{O}_{3}$ : 490.2814 .

3-[4-Methyl-2-(morpholin-4-yl)pyrimidine-5-carbonyl]6,8,8,9-tetramethyl-6,7,8,9-tetrahydro- $\mathrm{H}$-pyrano[3,2-g]quinolin-2-one (5e). The yield was $0.86 \mathrm{~g}(93 \%)$, m.p. $208-210{ }^{\circ} \mathrm{C}$. ${ }^{1} \mathrm{H}$ NMR, $\delta: 1.25$ (s, $\left.3 \mathrm{H}, \mathrm{C}(8) \mathrm{CH}_{3}\right) ; 1.28-1.32\left(\mathrm{~m}, 6 \mathrm{H}, \mathrm{C}(6) \mathrm{CH}_{3}\right.$, $\left.\mathrm{C}(8) \mathrm{CH}_{3}\right) ; 1.41(\mathrm{t}, 1 \mathrm{H}, \mathrm{H}(7), J=13.0 \mathrm{~Hz}) ; 1.88(\mathrm{dd}, 1 \mathrm{H}, \mathrm{H}(7)$, $J=13.0 \mathrm{~Hz}, J=4.0 \mathrm{~Hz}) ; 2.41\left(\mathrm{~s}, 3 \mathrm{H}, \mathrm{N}(9) \mathrm{CH}_{3}\right) ; 2.78(\mathrm{~m}, 1 \mathrm{H}$, $\mathrm{H}(6)) ; 2.92$ (s, $\left.3 \mathrm{H}, \mathrm{C}\left(4^{\prime}\right) \mathrm{CH}_{3}\right) ; 3.65$ (t, $4 \mathrm{H}, 2 \mathrm{NCH}_{2} \mathrm{CH}_{2} \mathrm{O}$, $J=4.9 \mathrm{~Hz}) ; 3.80\left(\mathrm{t}, 4 \mathrm{H}, 2 \mathrm{NC}_{2} \mathrm{CH}_{2} \mathrm{O}, J=4.9 \mathrm{~Hz}\right) ; 6.42$ (s, $1 \mathrm{H}, \mathrm{H}(10)) ; 7.49$ (s, $1 \mathrm{H}, \mathrm{H}(5)) ; 8.30$ (s, $1 \mathrm{H}, \mathrm{H}(4))$; 8.41 (s, $\left.1 \mathrm{H}, \mathrm{H}\left(2^{\prime}\right)\right) .{ }^{13} \mathrm{C}$ NMR, $\delta: 19.4 ; 24.1 ; 25.5 ; 26.7 ; 29.0 ; 32.7 ; 44.2$; $45.3 ; 56.2 ; 66.4 ; 96.5 ; 107.9 ; 117.3 ; 121.4 ; 126.5 ; 126.9 ; 148.7$; $152.2 ; 157.2 ; 159.8 ; 160.4 ; 167.9 ; 189.8$. MS, found: $m / z 463.2343$ $[\mathrm{M}+\mathrm{H}]^{+}$, calculated for $\mathrm{C}_{26} \mathrm{H}_{30} \mathrm{~N}_{4} \mathrm{O}_{4}: 463.2341$.

3-[2-[(4-Methylpyrimidine-2-yl)thio]acetyl]-6,8,8,9-tetramethyl-8,9-dihydro-2H-pyrano[3,2-g]quinolin-2-one (7a). The yield was $0.71 \mathrm{~g}(84 \%)$, m.p. $173-175{ }^{\circ} \mathrm{C} .{ }^{1} \mathrm{H}$ NMR, $\delta: 1.37$ (s, $\left.6 \mathrm{H}, \mathrm{C}(8)\left(\mathrm{CH}_{3}\right)_{2}\right) ; 1.92\left(\mathrm{~s}, 3 \mathrm{H}, \mathrm{C}(6) \mathrm{CH}_{3}\right) ; 2.31(\mathrm{~s}, 3 \mathrm{H}$, $\left.\mathrm{N}(9) \mathrm{CH}_{3}\right) ; 2.93\left(\mathrm{~s}, 3 \mathrm{H}, \mathrm{C}\left(4^{\prime}\right) \mathrm{CH}_{3}\right) ; 4.65\left(\mathrm{~s}, 2 \mathrm{H}, \mathrm{CH}_{2}\right) ; 5.50$ (s, $1 \mathrm{H}, \mathrm{H}(7)) ; 6.43$ (s, $1 \mathrm{H}, \mathrm{H}(10)) ; 7.03$ (d, $1 \mathrm{H}, \mathrm{H}\left(6^{\prime}\right)$, $J=5.1 \mathrm{~Hz}) ; 7.43(\mathrm{~s}, 1 \mathrm{H}, \mathrm{H}(5)) ; 8.40\left(\mathrm{~d}, 1 \mathrm{H}, \mathrm{H}\left(5^{\prime}\right), J=5.1 \mathrm{~Hz}\right)$; $8.50(\mathrm{~s}, 1 \mathrm{H}, \mathrm{H}(4)) .{ }^{13} \mathrm{C}$ NMR, $\delta: 18.6 ; 23.9 ; 29.2 ; 32.4 ; 41.4$; 58.8 ; $95.6 ; 108.2 ; 114.4 ; 117.1 ; 120.3 ; 125.5 ; 130.7 ; 148.6 ; 151.7$; $155.9 ; 157.5 ; 159.1 ; 160.3 ; 167.8 ; 170.4 ; 190.7$. MS, found: $m / z$ $422.1534[\mathrm{M}+\mathrm{H}]^{+}$, calculated for $\mathrm{C}_{23} \mathrm{H}_{23} \mathrm{~N}_{3} \mathrm{O}_{3} \mathrm{~S}: 422.1534$.

3-[2-[(4-Methyl-4H-1,2,4-triazol-3-yl)thio]acetyl]-6,8,8,9tetramethyl-8,9-dihydro-2H-pyrano[3,2-g]quinolin-2-one (7b). The yield was $0.67 \mathrm{~g}(82 \%)$, m.p. $238-240{ }^{\circ} \mathrm{C} .{ }^{1} \mathrm{H}$ NMR, $\delta: 1.37$ (s, $\left.6 \mathrm{H}, \mathrm{C}(8)\left(\mathrm{CH}_{3}\right)_{2}\right) ; 1.93\left(\mathrm{~s}, 3 \mathrm{H}, \mathrm{C}(6) \mathrm{CH}_{3}\right) ; 2.94(\mathrm{~s}, 3 \mathrm{H}$, $\left.\mathrm{NCH}_{3}\right) ; 3.58\left(\mathrm{~s}, 3 \mathrm{H}, \mathrm{N}\left(4^{\prime}\right) \mathrm{CH}_{3}\right) ; 4.64\left(\mathrm{~s}, 2 \mathrm{H}, \mathrm{CH}_{2}\right) ; 5.51$ 
(s, $1 \mathrm{H}, \mathrm{H}(7)) ; 6.43$ (s, $1 \mathrm{H}, \mathrm{H}(10)) ; 7.43$ (s, $1 \mathrm{H}, \mathrm{H}(5))$; 8.51 (s, $\left.1 \mathrm{H}, \mathrm{H}\left(5^{\prime}\right)\right) ; 8.52$ (s, $\left.1 \mathrm{H}, \mathrm{H}(4)\right) .{ }^{13} \mathrm{C}$ NMR, $\delta: 18.6$; $29.2 ; 31.2 ; 32.5 ; 44.7 ; 58.9 ; 95.6 ; 108.3 ; 113.3 ; 120.4 ; 125.1$; $130.8 ; 146.6 ; 148.9 ; 149.1 ; 149.2 ; 151.9 ; 159.3 ; 160.2 ; 189.9 . \mathrm{MS}$, found: $m / z 411.1483[\mathrm{M}+\mathrm{H}]^{+}$, calculated for $\mathrm{C}_{21} \mathrm{H}_{22} \mathrm{~N}_{4} \mathrm{O}_{3} \mathrm{~S}$ : 411.1487

3-[2-[(4-Methylpyrimidin-2-yl)thio]acetyl]-6,8,8,9-tetramethyl-6,7,8,9-tetrahydro-2H-pyrano[3,2-g]quinolin-2-one (8a). The yield was $0.67 \mathrm{~g}(79 \%)$, m.p. $167-168{ }^{\circ} \mathrm{C} .{ }^{1} \mathrm{H}$ NMR, $\delta: 1.25$ $\left(\mathrm{s}, 3 \mathrm{H}, \mathrm{C}(8) \mathrm{CH}_{3}\right) ; 1.29\left(\mathrm{~d}, 3 \mathrm{H}, \mathrm{C}(6) \mathrm{CH}_{3}, J=6.6 \mathrm{~Hz}\right) ; 1.31$ (s, $\left.3 \mathrm{H}, \mathrm{C}(8) \mathrm{CH}_{3}\right) ; 1.40$ (t, $\left.1 \mathrm{H}, \mathrm{H}(7), J=13.1 \mathrm{~Hz}\right) ; 1.87$ (dd, $1 \mathrm{H}$, $\mathrm{H}(7), J=13.1 \mathrm{~Hz}, J=3.9 \mathrm{~Hz}) ; 2.31\left(\mathrm{~s}, 3 \mathrm{H}, \mathrm{N}(9) \mathrm{CH}_{3}\right) ; 2.78$ (m, $1 \mathrm{H}, \mathrm{H}(6)) ; 4.65$ (s, $\left.2 \mathrm{H}, \mathrm{CH}_{2}\right) ; 6.46$ (s, $\left.1 \mathrm{H}, \mathrm{H}(10)\right) ; 7.03$ $\left(\mathrm{d}, 1 \mathrm{H}, \mathrm{H}\left(6^{\prime}\right), J=5.1 \mathrm{~Hz}\right) ; 7.52(\mathrm{~s}, 1 \mathrm{H}, \mathrm{H}(5)) ; 8.39$ (d, $1 \mathrm{H}$, $\mathrm{H}\left(5^{\prime}\right), J=5.1 \mathrm{~Hz}$ ); 8.52 (s, $\left.1 \mathrm{H}, \mathrm{H}(4)\right) .{ }^{13} \mathrm{C}$ NMR, $\delta$ : 19.3; 23.9; $25.5 ; 28.9 ; 32.8 ; 41.4 ; 45.1 ; 56.5 ; 96.2 ; 107.9 ; 114.2 ; 117.1 ; 127.1$; $127.3 ; 148.7 ; 152.8 ; 157.5 ; 157.7 ; 150.5 ; 167.8 ; 170.4 ; 190.8 . \mathrm{MS}$, found: $m / z 424.1690[\mathrm{M}+\mathrm{H}]^{+}$, calculated for $\mathrm{C}_{23} \mathrm{H}_{25} \mathrm{~N}_{3} \mathrm{O}_{3} \mathrm{~S}$ : 424.1691

3-[2-[(4-Methyl-4H-1,2,4-triazol-3-yl)thio]acetyl]-6,8,8,9tetramethyl-6,7,8,9-tetrahydro- $2 \mathrm{H}$-pyrano[3,2-g]quinolin-2-one (8b). The yield was $0.66 \mathrm{~g}(80 \%)$, m.p. $209-211^{\circ} \mathrm{C} .{ }^{1} \mathrm{H}$ NMR, $\delta$ : $1.26\left(\mathrm{~s}, 3 \mathrm{H}, \mathrm{C}(8) \mathrm{CH}_{3}\right) ; 1.28-1.32\left(\mathrm{~m}, 6 \mathrm{H}, \mathrm{C}(6) \mathrm{CH}_{3}\right.$, $\left.\mathrm{C}(8) \mathrm{CH}_{3}\right) ; 1.40(\mathrm{t}, 1 \mathrm{H}, \mathrm{H}(7), J=13.2 \mathrm{~Hz}) ; 1.87(\mathrm{dd}, 1 \mathrm{H}, \mathrm{H}(7)$, $J=13.2 \mathrm{~Hz}, J=4.0 \mathrm{~Hz}) ; 2.76(\mathrm{~m}, 1 \mathrm{H}, \mathrm{H}(6)) ; 2.93(\mathrm{~s}, 3 \mathrm{H}$, $\left.\mathrm{N}(9) \mathrm{CH}_{3}\right) ; 3.58\left(\mathrm{~s}, 3 \mathrm{H}, \mathrm{N}\left(4^{\prime}\right) \mathrm{CH}_{3}\right) ; 4.64\left(\mathrm{~s}, 2 \mathrm{H}, \mathrm{CH}_{2}\right) ; 6.45$ (s, $1 \mathrm{H}, \mathrm{H}(10)) ; 7.51$ (s, $1 \mathrm{H}, \mathrm{H}(5)) ; 8.51$ (s, $1 \mathrm{H}, \mathrm{H}\left(5^{\prime}\right)$ ); 8.53 (s, $1 \mathrm{H}, \mathrm{H}(4)) .{ }^{13} \mathrm{C}$ NMR, $\delta: 19.3 ; 25.5 ; 26.6 ; 28.9 ; 31.2 ; 32.9$; $44.6 ; 45.0 ; 56.6 ; 96.2 ; 108.0 ; 113.0 ; 127.2 ; 127.4 ; 146.5 ; 149.0$; $149.2 ; 153.1 ; 157.8 ; 160.5 ; 190.0$. MS, found: $m / z 413.1641$ $[\mathrm{M}+\mathrm{H}]^{+}$, calculated for $\mathrm{C}_{21} \mathrm{H}_{24} \mathrm{~N}_{4} \mathrm{O}_{3} \mathrm{~S}: 413.1643$.

Inhibitory activity of compounds $4 a-c, 5 a-e, 7 a, b$, and $8 a, b$ against blood coagulation factors Xa and XIa. The inhibition of blood clotting factors Xa and XIa by the synthesized compounds was studied by measuring the kinetics of hydrolysis of substrates specific for each of these enzymes in the presence of these compounds. A specific low-molecular-weight chromogenic substrate S2765 (Z-D-Arg-Gly-Arg-pNA - 2HCl) was used in the case of factor Xa, while the substrate S2366 (pyroGlu-Pro-ArgpNA $\cdot \mathrm{HCl}$ ) (both from Chromogenix, USA) was used for factor XIa.

A buffer containing $140 \mathrm{~m} M \mathrm{NaCl}, 20 \mathrm{~m} M$ HEPES, and $0.1 \%$ PEG 6000 (pH 8.0) was placed in the wells of a 96-well plate, followed by the addition of factor Xa (final concentration $2.5 \mathrm{nmol} \mathrm{L}{ }^{-1}$ ) or XIa (final concentration $0.8 \mathrm{nmol} \mathrm{L}^{-1}$ ), the substrate S2765 (final concentration $200 \mu \mathrm{mol} \mathrm{L}{ }^{-1}$ ) or S2366 (final concentration $200 \mu \mathrm{mol} \mathrm{L} \mathrm{L}^{-1}$ ), and a solution of the test compound in DMSO (final concentration $30 \mu \mathrm{mol} \mathrm{L} \mathrm{L}^{-1}$, the DMSO content in the well was no more than $2 \%$ ). The kinetics of the formation of 4-nitroaniline was measured using a THERMOmax Microplate Reader (Molecular Devices Corporation, USA) by the absorption of light with a wavelength of $405 \mathrm{~nm}$. The initial rate of substrate degradation was determined from the initial slope of the 4-nitroaniline formation curve. The rate of substrate degradation by the enzyme in the presence of the inhibitor was expressed as a percentage relative to the rate of substrate degradation in the absence of the inhibitor. The results are presented in Table 1. The data obtained were processed using the GraphPad Prism and OriginPro 8 software.

This work was financially supported by the Russian Science Foundation (Project No. 18-74-10097).
This work does not involve human participants and animal subjects.

The authors declare no competing interests.

\section{References}

1. Y. Abdulsattar, R. Bhambri, A. Nogid, Pharmacy Therapeutics, $2009, \mathbf{3 4}, 238$.

2. C. Frost, J. Wang, S. Nepal, A. Schuster, Y. C. Barrett, R. Mosqueda-Garcia, R. A. Reeves, F. LaCreta, Br. J. Clin. Pharmacol., 2013, 75, 476; DOI: 10.1111/j.1365-2125.2012.04369.x.

3. T. Furugohri, K. Isobe, Y. Honda, C. Kamisato-Matsumoto, N. Sugiyama, T. Nagahara, Y. Morishima, T. Shibano, J. Thromb. Haemostasis, 2008, 6, 1542; DOI: 10.1111/j.15387836.2008.03064.x.

4. D. Ostrovsky, M. Udier-Blagovic, W. L. Jorgensen, J. Med. Chem., 2003, 46, 5691; DOI: 10.1021/jm030288d.

5. A. G. G. Turpie, Arterioscler. Thromb., Vasc. Biol., 2007, 27, 1238; DOI: 10.1161/ATVBAHA.107.139402.

6. J. M. Walenga, W. P. Jeske, D. Hoppensteadt, J. Fareed, Curr. Opin. Invest. Drugs, 2003, 4, 272.

7. K. A. Bauer, J. Thromb. Thrombolysis, 2006, 21, 67; DOI: 10.1007/s11239-006-5579-4.

8. M. L. Quan, P. C. Wong, C. Wang, F. Woerner, J. M. Smallheer, F. A. Barbera, J. M. Bozarth, R. L. Brown, M. R. Harpel, J. M. Luettgen, P. E. Morin, T. Peterson, V. Ramamurthy, A. R. Rendina, K. A. Rossi, C. A. Watson, A. Wei, G. Zhang, D. Seiffert, R. R. Wexler, J. Med. Chem., 2014, 57, 955; DOI: $10.1021 / \mathrm{jm} 401670 x$.

9. P. C. Wong, M. L. Quan, C. A. Watson, E. J. Crain, M. R. Harpel, A. R. Rendina, J. M. Luettgen, R. R. Wexler, W. A. Schumacher, D. A. Seiffert, J. Thromb. Thrombolysis, 2015, 40, 416; DOI: 10.1007/s11239-015-1258-7.

10. D. J. P. Pinto, M. J. Orwat, L. M. Smith, M. L. Quan, P. Y. S. Lam, K. A. Rossi, A. Apedo, J. M. Bozarth, Y. Wu, J. J. Zheng, B. Xin, N. Toussaint, P. Stetsko, O. Gudmundsson, B. Maxwell, E. J. Crain, P. C. Wong, Z. Lou, T. W. Harper, S. A. Chacko, J. E. Myers, Jr., S. Sheriff, H. Zhang, X. Hou, A. Mathur, D. A. Seiffert, R. R. Wexler, J. M. Luettgen, W. R. Ewing, J. Med. Chem., 2017, 60, 9703; DOI: 10.1021/ acs.jmedchem.7b01171.

11. D. J. P. Pinto, J. M. Smallheer, J. R. Corte, E. J. D. Austin, C. Wang, T. Fang, L. M. Smith II, K. A. Rossi, A. R. Rendina, J. M. Bozarth, G. Zhang, A. Wei, V. Ramamurthy, S. Sheriff, J. E. Myers, Jr., P. E. Morin, J. M. Luettgen, D. A. Seiffert, M. L. Quan, R. R. Wexler, Bioorg. Med. Chem. Lett., 2015, 25, 1635; DOI: 10.1016/j.bmcl.2015.01.028.

12. Z. Hu, C. Wang, W. Han, K. A. Rossi, J. M. Bozarth, Y. Wu, S. Sheriff, J. E. Myers, Jr., J. M. Luettgen, D. A. Seiffert, R. R. Wexler, M. L. Quan, Bioorg. Med. Chem. Lett., 2018, 28, 987; DOI: 10.1016/j.bmcl.2018.02.049.

13. J. R. Corte, T. Fang, D. J. P. Pinto, M. J. Orwat, A. R. Rendina, J. M. Luettgen, K. A. Rossi, A. Wei, V. Ramamurthy, J. E. Myers, Jr., S. Sheriff, R. Narayanan, T. W. Harper, J. J. Zheng, Y.-X. Li, D. A. Seiffert, R. R. Wexler, M. L. Quan, Bioorg. Med. Chem., 2016, 24, 2257; DOI: 10.1016/ j.bmc. 2016.03.062.

14. A. J. Obaidullah, R. A. Al-Horani, Cardiovasc. Hematol. Agents Med.Chem., 2017, 15, 40; DOI: 10.2174/1871525715666 170529093938 . 
15. M. L. Quan, D. J. P. Pinto, J. M. Smallheer, W. R. Ewing, K. A. Rossi, J. M. Luettgen, D. A. Seiffert, R. R. Wexler, J. Med. Chem., 2018; 61, 7425; DOI: 10.1021/acs.jmedchem. $8 \mathrm{~b} 00173$.

16. S. Maignan, V. Mikol, Curr. Top. Med. Chem., 2001, 1, 161; DOI: $10.2174 / 1568026013395461$.

17. S. Roehrig, A. Straub, J. Pohlmann, T. Lampe, J. Pernerstorfer, K. H. Schlemmer, P. Reinemer, E. Perzborn, J. Med. Chem., 2005, 48, 5900; DOI: 10.1021/jm050101d.

18. K. M. Amin, N. M. A. Gawad, D. E. A. Rahman, M. K. E. Ashry, Bioorg. Chem., 2014, 52, 31; DOI: 10.1016/j.bioorg. 2013.11.002.

19. S. Verespy III, A. Y. Mehta, D. Afosah, R. A. Al-Horani, U. R. Desai, Sci. Rep., 2016, 6, 24043, DOI: 10.1038/srep24043.

20. M. S. Buchanan, A. R. Carroll, D. Wessling, M. Jobling, V. M. Avery, R. A. Davis, Y. Feng, Y. Xue, L. Öster, T. Fex, J. Deinum, J. N. A. Hooper, R. J. Quinn, J. Med. Chem., 2008, 51, 3583; DOI: 10.1021/jm800314b.

21. O. Fjellström, S. Akkaya, H. G. Beisel, P. O. Eriksson, K. Erixon, D. Gustafsson, U. Jurva, D. Kang, D. Karis, W. Knecht, V. Nerme, I. Nilsson, T. Olsson, A. Redzic, R. Roth, J. Sandmark, A. Tigerstrom, L. Oster, PLOS One, 2015, 10, e0113705; DOI: 10.1371/journal.pone.0113705.

22. F. Santana-Romo, C. F. Lagos, Y. Duarte, F. Castillo, Y. Moglie, M. A. Maestro, N. Charbe, F. C. Zacconi, Molecules, 2020, 25, 491; DOI: 10.3390/molecules25030491.

23. G. Wissel, P. Kudryavtsev, L. Ghemtio, P. Tammela, P. Wipf, M. Yliperttula, M. Finel, A. Urtti, H. Kidron, H. Xhaard, Bioorg. Med. Chem., 2015, 23, 3513; DOI: 10.1016/j.bmc. 2015.04.029.

24. F. Gao, L. Ye, Y. Wang, F. Kong, Sh. Zhao, J. Xiao, G. Huang, Eur. J. Med. Chem., 2019, 183, 111678; DOI: 10.1016/ j.ejmech.2019.111678.

25. V. Kartsev, Kh. S. Shikhaliev, A. Geronikaki, S. M. Medvedeva, I. V. Ledenyova, M. Yu. Krysin, A. Petrou, A. Ciric, J. Glamoclija, M. Sokovic, Eur. J. Med. Chem., 2019, 175, 201; DOI: 10.1016/j.ejmech.2019.04.046.

26. J. Gao, Z. Zhanga, B. Zhanga, Q. Maoa, X. Daia, Q. Zoub, Y. Leia, Y. Fenga, S. Wang, Bioorg. Chem., 2020, 95, 103564; DOI: 10.1016/j.bioorg.2019.103564.

27. N. Novichikhina, I. Ilin, A. Tashchilova, A. Sulimov, D. Kutov, I. Ledenyova, M. Krysin, Kh. Shikhaliev, A. Gantseva,
E. Gantseva, N. Podoplelova, V. Sulimov, Molecules, 2020, 25, 1889; DOI: 10.3390/molecules25081889.

28. A. Djemoui, A. Naouri, M. R. Ouahrani, D. Djemoui, S. Lahcene, M. B. Lahrech, L. Boukenna, H. M. T. Albuquerque, L. Saher, D. H. A. Rocha, F. L. Monteiro, L. A. Helguero, K. Bachari, O. Talhi, A. M. S. Silva, J. Mol. Struct., 2020, 1204, 127487; DOI: 10.1016/j.molstruc. 2019.127487

29. N. P. Novichikhina, A. S. Shestakov, A. Yu. Potapov, E. A. Kosheleva, G. V. Shatalov, V. N. Verezhnikov, D. Yu. Vandyshev, I. V. Ledeneva, Kh. S. Shikhaliev, Russ. Chem. Bull., 2020, 69, 787; DOI: 10.1007/s11172-020-2834-3.

30. J. Shi, N. Luo, M. Ding, X. Bao, Chin. Chem. Lett., 2020, 31, 434; DOI: 10.1016/j.cclet.2019.06.037.

31. S. Li, D. Cao, Z. Hu, Z. Li, X. Meng, X. Han, W. Ma, Chem. Heterocycl. Compd., 2020, 56, 219; DOI: 10.1007/s10593020-02647-y.

32. G. Sakaine, G. Smits, P. Arsenyan, Chem. Heterocycl. Compd., 2020, 56, 572; DOI: 10.1007/s10593-020-02702-8.

33. D. Khan, S. Mukhtar, M. A. Alsharif, M. I. Alahmdi, N. Ahmed, Tetrahedron Lett., 2017, 58, 3183; DOI: 10.1016/ j.tetlet.2017.07.018.

34. C. Ranjith, N. Paul, K. K. Vijayan, Asian J. Chem., 2011, 23, 235.

35. P. Verdia, F. Santamarta, E. Tojo, Molecules, 2011, 16, 4379; DOI: $10.3390 /$ molecules 16064379 .

36. X. He, Y. Shang, Y. Zhou, Zh. Yu, G. Han, W. Jin, J. Chen, Tetrahedron, 2015, 71, 863; DOI: 10.1016/j.tet.2014. 12.042.

37. A. Yu. Potapov, D. Yu. Vandyshev, Y. Refki, I. V. Ledenyova, O. V. Ovchinnikov, M. S. Smirnov, Kh. S. Shikhaliev, Russ. J. Gen. Chem., 2020, 90, 1216; DOI: 10.1134/ S1070363220070075.

38. G. M. Manahelohe, A. Yu. Potapov, Kh. S. Shikhaliev, Russ. Chem. Bull., 2016, 65, 1145; DOI: 10.1007/s11172-016-1427-7.

Received December 2, 2020; in revised form December 23, 2020; accepted January14, 2021 